\section{Q Fever Endocarditis and a New Genotype of Coxiella burnetii, Greece}

loulia Karageorgou, Nektarios Kogerakis, Stavroula Labropoulou, Sophia Hatzianastasiou, Andreas Mentis, George Stavridis, Emmanouil Angelakis

Author affiliations: Hellenic Pasteur Institute, Athens, Greece (I. Karageorgou, S. Labropoulou, A. Mentis, E. Angelakis); Onassis Cardiac Surgery Center, Athens (N. Kogerakis, S. Hatzianastasiou, G. Stavridis); Aix Marseille Université, Marseille, France (E. Angelakis)

DOI: https://doi.org/10.3201/eid2610.191616
Underdiagnosis of Coxiella burnetii infections in Greece is possible because of lack of awareness by physicians, and most suspected cases are in patients with no bovine contact. We found serologic evidence of C. burnetii infection throughout Greece and identified a new $C$. burnetii genotype in the aortic valve of a patient with $Q$ fever endocarditis.

Q fever is a worldwide zoonosis caused by an obligate intracellular bacterium, Coxiella burnetii $(1,2)$. Although the classification of C. burnetii by the Centers for Disease Control and Prevention (Atlanta, GA, USA) as a potential bioterrorism agent resulted in the disease becoming reportable in many countries (3), Q fever is not considered a public health problem in Greece, and few cases have been recorded (3).

\title{
etymologia
}

Mimivirus [mĭm'î-vī'rəs]

Clyde Partin

Tf virus (Latin: slimy) challenges the definition of what constitutes life, Ithe DNA mimivirus tests how we define virus. This unidentifiable "bacterium" infecting Acanthamoeba polyphaga, was isolated in 1992 from a hospital cooling tower in Bradford, England. Thus, the original name was Bradfordcoccus, and it was considered a culprit for a pneumonia outbreak at this hospital.

Researchers brought samples to Didier Raoult and colleagues at Aix-Marseille University, who eventually identified this "bacterium" as a novel virus in 2003. The physical size, genomic content, and ability of the outer protein coat to stain gram positive, thus mimicking (Latin: imitate) prokaryotic bacteria, indicated that this pathogen might be a bacterium.

Raoult initially claimed that the moniker meant "mimicking microbe" but later sheepishly recounted a childhood memory about his father, a physician-scientist, who created stories to explain evolution. Featured prominently in these whimsical narratives was an anthropomorphic character named "Mimi the amoeba."

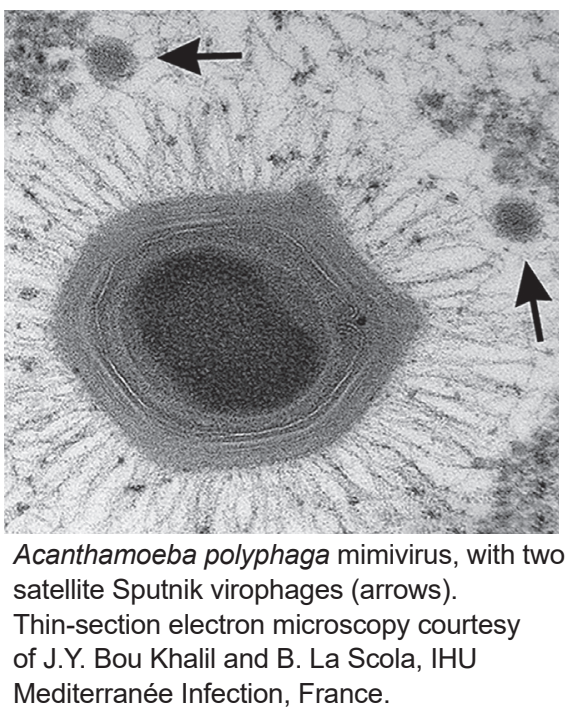

\section{Sources}

1. Redefining life [cited 2020 Jul 2]. https:/ / www.rsb.org. uk/biologist-features/158-biologist/features/1490-largerthan-life

2. Viruses reconsidered [cited $2020 \mathrm{Jul}$ 20]. https://www. the-scientist.com/features/viruses-reconsidered37867

Author affiliation: Emory University School of Medicine, Atlanta, Georgia, USA

Address for correspondence: Clyde Partin, Emory University School of Medicine, 1365 Clifton Rd NE, Clinic A, 1st Fl, Atlanta, GA 30322, USA; email: wpart01@emory.edu

DOI: https://doi.org/10.3201/eid2610.ET2610 
Our referent laboratory for the diagnosis of $Q$ fever was deployed in the Hellenic Pasteur Institute in February 2019. We tested serum samples from all patients by using an immunofluorescence assay (IFA) for C. burnetii phase I and II antigens as described $(4,5)$. Patients are classified as having acute Q fever; persistent, focalized C. burnetii infection; or evidence of past infection (6). Moreover, anticardiolipin IgG is routinely measured for patients given a diagnosis of acute $Q$ fever (6).

During the first 7 months of testing, we received 209 serum samples from patients suspected of having $Q$ fever. We provided diagnoses of acute $Q$ fever for $1(0.5 \%)$ patient and persistent $C$. burnetii focalized endocarditis for $2(1.0 \%)$ patients; $12(6.0 \%)$ patients showed evidence of $C$. burnetii infection. The patient given a diagnosis of acute $Q$ fever also had high levels of anticardiolipin IgG (>140 GPLU). Further investigation also showed large, transient, aortic vegetation. Thus, this patient was considered as possibly having acute $Q$ fever endocarditis $(4,7)$, but contact with the patient was lost.

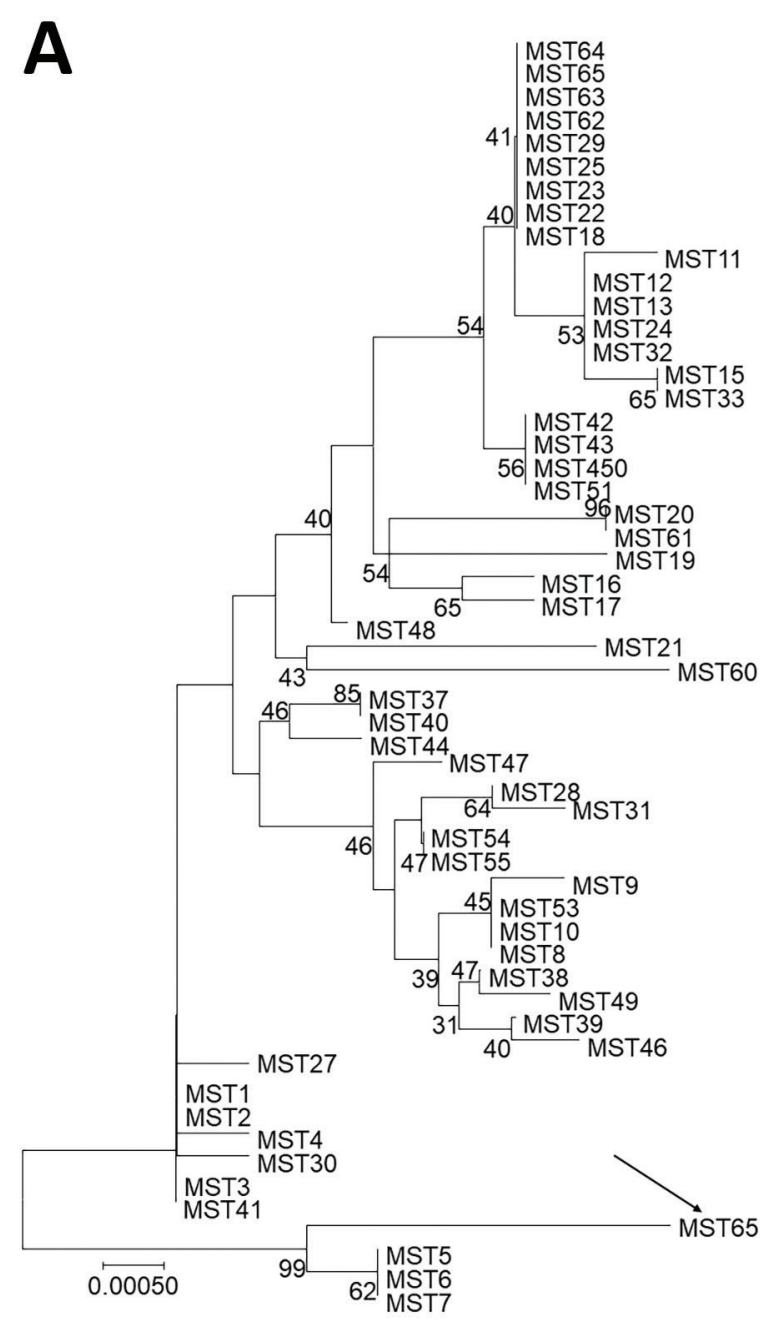

Epidemiologic information was obtained for 102 patients, including all patients with a positive IFA result for $C$. burnettii. This information showed that only $22 \%$ of these patients reported previous contact with bovids. Most patients reported a previous tick bite $(35 \%)$; contact with cats $(16 \%)$, dogs $(7 \%)$, rats $(4 \%)$, or other animals $(7 \%)$. In addition, $9 \%$ of these patients reported no animal contact.

We provide a detailed history for 1 patient given a diagnosis of persistent $C$. burnetii focalized endocarditis. A 45-year-old sheepherder, a resident of a rural area in southern Greece, came to the local district hospital with a 2-week history of spiking fevers, peripheral edema, and night sweats. He reported nonspecific symptoms gradually leading to anorexia and debilitating weakness for the previous year.

Cardiac ultrasound showed a severely regurgitant bicuspid aortic valve, a paravalvular abscess $(2.6$ $\mathrm{cm} \times 1.6 \mathrm{~cm})$, aortic root dilatation $(5.3 \mathrm{~cm})$, and vegetations. Cardiac computed tomography confirmed the ultrasound findings. IFA results were positive for

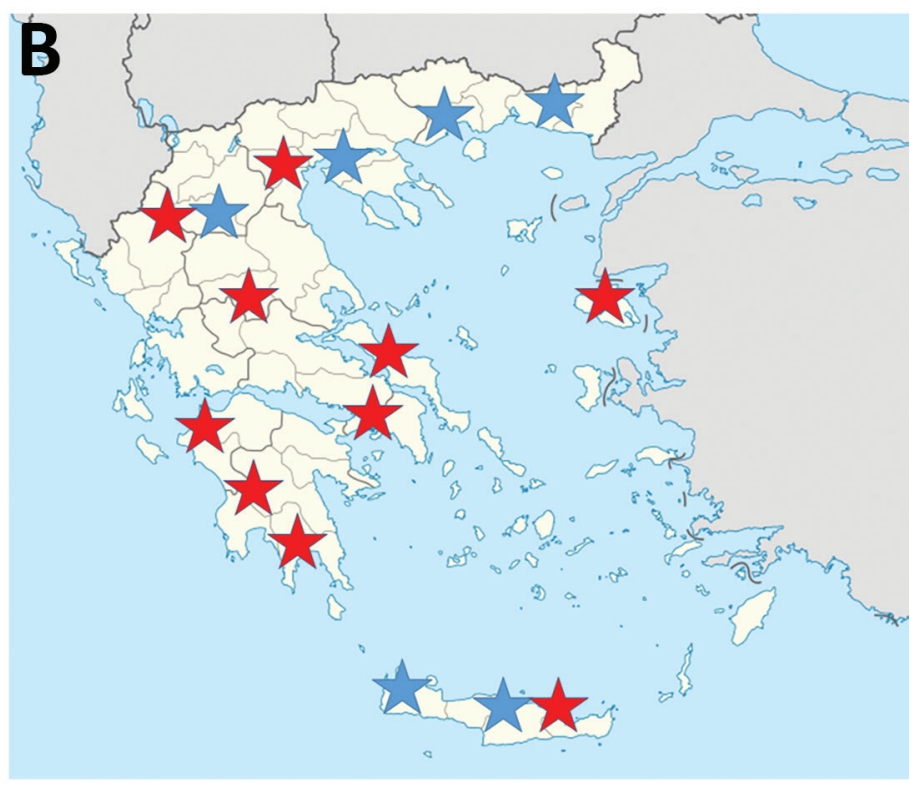

Figure. Investigation of Q fever endocarditis, Greece. A) Neighborjoining tree of Coxiella burnetii genotypes determined by multispacer sequence typing. Analysis was performed by using MEGA version 7 software (https://www.megasoftware.net) and the neighbor-joining method (maximum composite likelihood method) with 1,000 replicates. Numbers along branches are bootstrap values. Arrow indicates new genotype from Greece. Scale bar indicates nucleotide substitutions per site. B) Seroepidemiologic evidence of $C$. burnetii cases in Greece. Blue stars indicate previous studies, and red stars indicate this study. MST, mutispacer type. 
C. burnetii: phase I IgG titer 1:3,200, phase I IgM titer 0; and phase II IgG titer 1:3,200, phase II IgM titer 0 . This serum sample was negative for $C$. burnetii by real-time PCR for insertion sequence (IS) 1111 and the IS30A spacers (8). Thus, we provided a diagnosis of $C$. burnetii endocarditis, and the patient was transferred to a tertiary care center for surgical management.

The patient underwent an aortic root replacement (Bentall procedure) with pericardial composite graft after extensive debridement and reconstruction of the root with the use of autologous pericardium. His aortic valve was positive for C. burnetii for IS1111 and IS30A spacers by real-time PCR. Multispacer sequence typing (MST) was performed as described and consisted of 10 different spacers of the C. burnetii genome: Cox2, 5, 6, 18, 20, 22, 37, 51, 56, and 57 (5). We identified a new MST genotype (MST65) by using web-based MST database (http://ifr48.timone.univmrs.fr/MST_Coxiella/mst) (Figure, panel A).

The patient was given oral doxycycline $(100 \mathrm{mg}$ $2 \times / \mathrm{d})$ and hydroxychloroquine $(200 \mathrm{mg} 3 \times / \mathrm{d})$ for $\geq 24$ months (9). A convalescence-phase serum sample obtained after 6 months of treatment was positive for C. burnetii: phase I IgG titer 1:800, phase I IgM titer 0, and phase II IgG titer 1:800, phase II IgM 0.

Our preliminary data show that physicians in Greece are not familiar with $Q$ fever because most of the suspected cases were in patients without bovine contact. A limitation of our study was that culture was not performed because of the absence of a Biosafety Level 3 laboratory. The fact that we did not provide diagnoses of classic, acute $Q$ fever showed that C. burnetii infection is suspected mostly in culture-negative serious endocarditis case-patients. Moreover, we identified a new C. burnetii genotype in the aortic valve of a patient who had $Q$ fever endocarditis. Recently, it was found that $C$. burnetii genotype 32 is circulating in sheep and goat in 8 different areas of Greece (10). The clinical manifestations of $Q$ fever depend, at least in part, on the C. burnetii genotype (5). However, although acute clinical manifestations are strain-specific, all genotypes have been associated with endocarditis (5).

We raise the question of underdiagnosis of C. burnetii infections in Greece. Our data have affected local clinical practice because we found serologic evidence of C. burnetii infection throughout most of Greece (Figure, panel B).

\section{About the Author}

Dr. Karageorgou is a biologist and researcher at the Hellenic Pasteur Institute in Athens, Greece. Her primary research interest is zoonotic pathogens.

\section{References}

1. Angelakis E, Raoult D. Q fever. Vet Microbiol. 2010;140:297309. https:// doi.org/10.1016/j.vetmic.2009.07.016

2. Eldin C, Mélenotte C, Mediannikov O, Ghigo E, Million M, Edouard S, et al. From Q fever to Coxiella burnetii infection: a paradigm change. Clin Microbiol Rev. 2017;30:115-90. https://doi.org/10.1128/CMR.00045-16

3. Kokkini S, Chochlakis D, Vranakis I, Angelakis E, Tselentis Y, Gikas A, et al. Antibody kinetics in serological indication of chronic $Q$ fever: the Greek experience. Int J Infect Dis. 2013;17:e977-80. https:/ / doi.org/10.1016/ j.ijid.2013.04.010

4. Million M, Thuny F, Bardin N, Angelakis E, Edouard S, Bessis S, et al. Antiphospholipid antibody syndrome with alvular vegetations in acute $Q$ fever. Clin Infect Dis. 2016;62:537-44. https:/ / doi.org/10.1093/cid/civ956

5. Angelakis E, Million M, D'Amato F, Rouli L, Richet H, Stein A, et al. Q fever and pregnancy: disease, prevention, and strain specificity. Eur J Clin Microbiol Infect Dis. 2013;32:361-8. https:// doi.org/10.1007/s10096-012-1750-3

6. Melenotte C, Protopopescu C, Million M, Edouard S, Carrieri MP, Eldin C, et al. Clinical features and complications of Coxiella burnetii infections from the French National Reference Center for Q Fever. JAMA Netw Open. 2018;1:e181580. https://doi.org/10.1001/ jamanetworkopen.2018.1580

7. Melenotte C, Epelboin L, Million M, Hubert S, Monsec T, Djossou F, et al. Acute $Q$ fever endocarditis: a paradigm shift following the systematic use of transthoracic echocardiography during acute $Q$ fever. Clin Infect Dis. 2019;69:1987-95. https://doi.org/10.1093/cid/ciz120

8. Angelakis E, Mediannikov O, Socolovschi C, Mouffok N, Bassene H, Tall A, et al. Coxiella burnetii-positive PCR in febrile patients in rural and urban Africa. Int J Infect Dis. 2014;28:107-10. https://doi.org/10.1016/j.ijid.2014.05.029

9. Melenotte C, Million M, Raoult D. New insights in Coxiella burnetii infection: diagnosis and therapeutic update. Expert Rev Anti Infect Ther. 2020;18:75-86. https://doi.org/ 10.1080/14787210.2020.1699055

10. Chochlakis D, Santos AS, Giadinis ND, Papadopoulos D, Boubaris L, Kalaitzakis E, et al. Genotyping of Coxiella burnetii in sheep and goat abortion samples. BMC Microbiol. 2018;18:204. https://doi.org/10.1186/ s12866-018-1353-y

$\overline{\text { Address for correspondence: Emmanouil Angelakis, Aix Marseille }}$ Université, Institut de Recherche pour le Développement, Assistance Publique Hôpitaux de Marseille, Vecteurs Infections Tropicales et Méditerranéennes, Institut Méditerranée Infection, 19-21 Blvd Jean Moulin, Marseille 13005, France; email: e.angelakis@hotmail.com 